\title{
Optically thick Herbig-Haro jets in photoionized regions
}

\author{
E. Masciadri ${ }^{1}$ and A. C. Raga ${ }^{2}$ \\ 1 Instituto de Astronomía, UNAM, Apartado Postal 70-264, 04510 D. F., México \\ e-mail: masciadri@astroscu. unam.mx \\ 2 Instituto de Ciencias Nucleares, UNAM, Apartado Postal 70-543, 04510 D. F., México
}

Received 23 May 2001 / Accepted 16 July 2001

\begin{abstract}
Over the last few years, a number of Herbig-Haro (HH) jets have been discovered within the confines of H II regions. These jets apparently are ejected from low mass stars that are similar to the sources of other "normal" HH jets, but the flows show some special characteristics which are a result of the photoionization due to the ionizing photons emitted by the star that excites the H II region. In this paper, we present HH jet models including an external, photoionizing radiation field, and study the effects of increasing ionizing photon fluxes $F_{*}$ on the structure of the head of the jet. We find that the morphology, the emission line fluxes and the line ratios change drastically as a function of increasing $F_{*}$. These results give general guidelines for deciding whether an $\mathrm{HH}$ jet actually does lie within an H II region, based on the morphology and spectroscopic characteristics of the object.
\end{abstract}

Key words. ISM: HH objects - hydrodynamics - methods: numerical

\section{Introduction}

An important, recent development in the study of HerbigHaro $(\mathrm{HH})$ objects has been the discovery of very well collimated $\mathrm{HH}$ jets which are immersed in phototoionized regions. The subject of externally photoionized $\mathrm{HH}$ jets was opened by Reipurth et al. (1998), who showed images and spectra of $4 \mathrm{HH}$ jets close to the bright rim illuminated by $\sigma$ Orionis. Several more such jets have now been discovered in the inner (Bally et al. 2000) and outer (Bally \& Reipurth 2001) regions of the Orion Nebula (M 42).

As far as we are aware, only two theoretical papers have been published on the subject of photoionized HH jets:

- Raga et al. (2000a) carried out a preliminary theoretical study applied to the HH 444 jet (see Reipurth et al. 1998). These authors presented numerical and analytic models of an initially neutral jet beam that becomes progressively photoionized as it travels into an $\mathrm{H}$ II region;

- Masciadri \& Raga (2001) carried out numerical simulations for modelling the HH 505 jet (see Bally \& Reipurth 2001). These authors focussed on the interaction between the jet and the expanding H II region, and treated the photoionization assuming that the flow is optically thin to the ionizing radiation.

Send offprint requests to: E. Masciadri,

e-mail: elena@astroscu.unam.mx
The present paper discusses the following idea. A HH jet immersed in an H II region can have neutral regions within the jet beam. For example, an initially neutral jet preserves a neutral region (in the side directed away from the photoionizing source) for at least part of the length of the jet beam. Raga et al. (2000a) concluded that for the parameters of $\mathrm{HH} 444$, the jet preserves a neutral region out to a distance of a few jet diameters away from the outflow source.

At larger distances from the source, where the jet beam has become fully ionized, the jet can still develop neutral regions. This effect can be obtained in the leading working surface (produced by the interaction of the head of the jet with the surrounding environment) or in internal working surfaces (produced as a result of a time-dependence of the ejection velocity), in which the density is strongly enhanced, and an ionization front can be trapped by the shocked jet (and/or environmental) material. Therefore, depending on the parameters of the jet, the working surfaces can develop neutral regions with trapped ionization fronts.

Jets with trapped ionization fronts show strong asymmetries in their radiative properties, having drastically different morphologies in maps of different emission lines. Such asymmetries are not observed in fully photoionized jets nor in jets which are not being photoionized by an external source.

In the present paper, we show the results from three numerical simulations of jets in the presence of external 
photoionizing fields of different intensities. These simulations illustrate the possible morphologies that are expected for the leading working surface of the jet as a function of increasing intensities of the external photoionizing field.

We discuss the parameters that we use for the numerical models in Sect. 2, and present the results from the simulations in Sect. 3. The observational properties of the resulting jet flows are discussed in Sect. 4 . Finally, the possible comparisons with observations of jets in photoionized regions are discussed in Sect. 5.

\section{Parameters for the jet models}

We propose to study the general effects of an external photoionizing radiative field on the leading working surface of an $\mathrm{HH}$ jet. For this study, we fix the jet parameters, and vary the strength of the impinging photon flux, so as to see the different possible effects of the external photoionization.

For the jet, we choose an initial jet velocity $v_{\mathrm{j}}=$ $200 \mathrm{~km} \mathrm{~s}^{-1}$, number density $n_{\mathrm{j}}=3000 \mathrm{~cm}^{-3}$ and temperature $T_{\mathrm{j}}=1000 \mathrm{~K}$. We assume that the jet has an initial top-hat, cylindrical cross section, with a $r_{\mathrm{j}}=7 \times 10^{15} \mathrm{~cm}$ radius. We furthermore assume that the jet is initially neutral.

For the surrounding, stationary environment, we assume an initial number density $n_{\mathrm{j}}=600 \mathrm{~cm}^{-3}$ and temperature $T_{\mathrm{j}}=10^{4} \mathrm{~K}$. We assume that hydrogen is initially fully ionized. In the first few timesteps, the temperature and the ionization fraction relax to the photoionization equilibrium with the impinging radiative field.

In the present paper, we discuss three jet simulations with the above parameters and with different external ionizing radiation fluxes. For the three models, we assume that the source of the ionizing radiation is far away from the jet (e.g., compared to the length of the jet), and that it lies in a direction perpendicular to the outflow axis. The remaining free parameter is the magnitude of the ionizing photon flux, for which we choose the three values listed in the second column of Table 1 . These three ionizing photon fluxes correspond to a source with an ionizing photon rate $S_{*}=7.25 \times 10^{48} \mathrm{~s}^{-1}$ (as expected, e.g., for $\theta^{1} \mathrm{C}$ Orionis) located at distances $d=10^{19}, 2 \times 10^{18}$ and $5 \times 10^{17} \mathrm{~cm}$ from the jet (third column, Table 1), provided that no absorptions occur in the intervening region. We assume that the spectrum emitted by the photoionizing source is a black body with $T_{\text {eff }}=35000 \mathrm{~K}$.

The results obtained from the three computed models are described in the following sections.

\section{Numerical simulations}

We have carried out numerical simulations of externally photoionized jets using the yguazú-a code. This code has a 3D, binary adaptive grid, employs the "flux-vector splitting" algorithm of Van Leer (1982), and has been described in detail by Raga et al. (2000b). We have included a continuity equation for neutral hydrogen (in order to follow the time-dependent ionization state of $\mathrm{H}$ ), and include the parametrized cooling function described by Raga et al. (1999).

The ionization state of $\mathrm{H}$ is computed considering radiative recombinations and photo- and collisional ionizations. The photoionization of $\mathrm{H}$ (and the corresponding heating rate) have been included in the single frequency approximation described by Cantó et al. (1998). The radiative transfer of ionizing photons (all of which are assumed to have frequencies close to the Lyman limit) is carried out with the "short characteristics" method which was implemented on a binary adaptive grid by Raga et al. (1997). The transfer of the diffuse ionizing photon field was not computed, and a "case B" radiative recombination coefficient was used for $\mathrm{H}$.

We should point out that the diffuse ionizing radiation field can in some cases have an important contribution to the photoionization of a dense region embedded in a photoionized nebula. This effect has been studied (in the context of dense clumps embedded in a nebula) by Cantó et al. (1998) and by Pavlakis et al. (2001). The photoionization of a jet by the diffuse ionizing field of the nebula would have the strongest possible effect in an ionization bounded nebula, in which the diffuse photon flux has a value of $\approx 1 / 6$ of the direct photon field from the photoionizing star (see Cantó et al. 1998). However, the objects in the $\sigma$ Orionis region (Reipurth et al. 1998) or the objects in the outer, SW region of M 42 (Bally \& Reipurth 2001) are clearly not in ionization bounded regions of a nebula, so that the diffuse ionizing field which is impinging on these jets is probably very low, and is not likely to have a strong effect.

The computations were carried out in a 4-level, binary adaptive grid with a maximum spatial resolution of $1.56 \times 10^{15} \mathrm{~cm}$, covering a $(1,1,2) \times 10^{17} \mathrm{~cm}$ domain (along the $x$-, $y$ - and $z$-axes, respectively). As the postbow shock cooling distance has a value of $\sim 10^{15} \mathrm{~cm}$ (as can be seen from the tabulation of plane-parallel shock models of Hartigan et al. 1987), it is only marginally resolved in the numerical simulations.

The initial jet cross section is centred at the $\left(x_{0}, y_{0}, z_{0}\right)=(3.75,5.0,0) \times 10^{16} \mathrm{~cm}$ position with respect to the origin of the computational domain, and the initial jet velocity is parallel to the $z$-axis. The ionizing photon source lies along the $x$-axis at a position $x=-d$, where the values of $d$ for the three computed models are given in Table 1.

Figure 1 shows the stratifications of the density and the hydrogen neutral fraction obtained after a $t=400 \mathrm{yr}$ time-integration for the three models. It is clear that while in model M1 (see Table 1) the beam of the jet remains mostly neutral, in the models M2 and M3 (with higher external ionizing photon fluxes) the jet beam is rapidly photoionized close to the injection point.

In model M1, only spatially limited regions show a low neutral fraction. These regions are a thin, post-bow shock layer, part of the cocoon of the jet, and the environmental 
Table 1. External ionizing photon fluxes, distance to the ionizing source, and peak $\mathrm{H} \alpha$ and $[\mathrm{O}$ I] 6300 intensities for the three models described in the text.

\begin{tabular}{ccccc}
\hline \hline Model & $\begin{array}{c}F_{*} \\
\left(\mathrm{~s}^{-1} \mathrm{~cm}^{-2}\right)\end{array}$ & $\begin{array}{c}d \\
(\mathrm{~cm})\end{array}$ & $\begin{array}{c}I_{\mathrm{H} \alpha} \\
\left(\mathrm{erg} \mathrm{s}^{-1} \mathrm{~cm}^{-2}\left({ }^{\prime \prime}\right)^{-2}\right)\end{array}$ \\
\hline \hline M1 & $5.76 \times 10^{9}$ & $10^{19}$ & $4.12 \times 10^{-14}$ & $8.01 \times 10^{-14}$ \\
M2 & $1.44 \times 10^{11}$ & $2 \times 10^{18}$ & $1.61 \times 10^{-12}$ & $1.38 \times 10^{-14}$ \\
M3 & $2.30 \times 10^{12}$ & $5 \times 10^{17}$ & $2.30 \times 10^{-12}$ & $1.62 \times 10^{-15}$ \\
\hline \hline
\end{tabular}

gas filling the region on the left of the computational domain. The impinging ionizing field does not have enough photons to photoionize the rest of the environmental gas within the domain. On the other hand, models M2 and M3 show an almost fully photoionized jet beam and surrounding environment (see Fig. 1).

In model M2, the high density gas within the working surface traps an ionization front. As a result of this, the post-bow shock and post-Mach disk gas can recombine, producing a partially neutral region (see Fig. 1). This neutral region in the working surface also shields the environmental gas (to the right of the jet) from the ionizing photon field (which impinges from the left), leading to the formation of a partially ionized "shadow region". This shadow region has interesting properties, but, as we shall see in the following section, it appears to be unobservable in any direct way.

In model M3, the impinging photon flux is strong enough to almost fully ionize the high density material within the working surface. Only a spatially very limited region with a hydrogen neutral fraction of $\approx 0.2$ survives along the right edge of the working surface. Only the faintest vestige of the "shadow" is seen in the environment to the right of the working surface.

Some of the same features can be seen in Fig. 2, which shows the temperature and optical depth (i.e., the optical depth at the Lyman limit to the direct radiation from the external, photoionizing source, due to the material within the computational domain) stratifications obtained for the three jet models after a $t=400 \mathrm{yr}$ time-integration (i.e., at the same time as Fig. 1). From this figure, it is clear that while the neutral regions have $T \approx 1000-2000 \mathrm{~K}$, the ionized regions have temperatures $\approx 5000 \mathrm{~K}$ (i.e., the temperature of the gas in photoionization equilibrium) or larger (in the regions in which there has been substantial shock heating).

Finally, from Fig. 2 it is also clear that the regions with substantial neutral hydrogen fractions lie completely within the boundaries of the region with $\tau>1$. While for model M1 the only region with $\tau<1$ is the left bow shock wing (and the nearby environment), for models M2 and M3 most of the computational domain is optically thin to the ionizing radiation.

\section{Expected observational properties}

In order to illustrate the observational properties of externally photoionized $\mathrm{HH}$ jet models, we have computed $\mathrm{H} \alpha$ and [O I] 6300 emission line maps from our three models (see Table 1). We have chosen these two emission lines because $\mathrm{H} \alpha$ is a line which is observed both in photoionized and in shock excited regions, and [O I] 6300 is a line which is strong in shocks, but very weak in photoionized regions. Therefore, we would expect drastically different behaviours for these two lines as a function of the external photoionizing flux impinging on the jet.

We have computed the $\mathrm{H} \alpha$ emission taking into consideration the recombination cascade and the collisional excitation from the ground state of $\mathrm{H}$, and the [O I] 6300 emission coefficient by solving the appropriate 5 -level atom problem, and assuming that the neutral fraction of oxygen follows the neutral hydrogen fraction (for a discussion of the atomic parameters that were used see Raga et al. 2001). We have then integrated the emission coefficient along lines of sight in order to obtain emission line maps, assuming that the $x z$-plane (i.e., the plane defined by the outflow axis and the direction to the ionizing photon source) lies on the plane of the sky.

The intensity maps computed in this way for the three models (see Table 1 ) at a $t=400 \mathrm{yr}$ integration time are shown in Fig. 3 (which shows the whole computational domain, and depicts the emission maps with a greyscale) and in Fig. 4 (which shows contour maps of the region around the leading working surface of the jet). The bow shock of the low photon flux model M1 shows an extended $\mathrm{H} \alpha$ wing in the $-x$ direction (i.e., towards the ionizing photon source), and a smaller extension of the [O I] 6300 emission along this bow shock wing (see Figs. 3 and 4). Also, the edge of the jet beam towards the photon source is faintly visible in the $\mathrm{H} \alpha$ map (see Fig. 3).

For model M2 (see Table 1), the $\mathrm{H} \alpha$ map becomes more symmetric, with two almost identical bow shock wings. However, in the region around the head of the bow shock, the trapped ionization front results in a strong asymmetry of the $\mathrm{H} \alpha$ emission (see Figs. 3 and 4). The [O I] 6300 emission shows a complete absence of emission along the far bow shock wings (a direct result of the fact that this region of the flow is completely photoionized), and is concentrated to the right side of the head of the bow shock (i.e., the side directed away from the ionizing photon source).

Finally, in model M3 (see Table 1) the $\mathrm{H} \alpha$ emission map is almost perfectly symmetrical, and only very faint [O I] 6300 emission is observed in the right hand side of the head of the bow shock. These results are consistent with the fact that for this model most of the flow is 


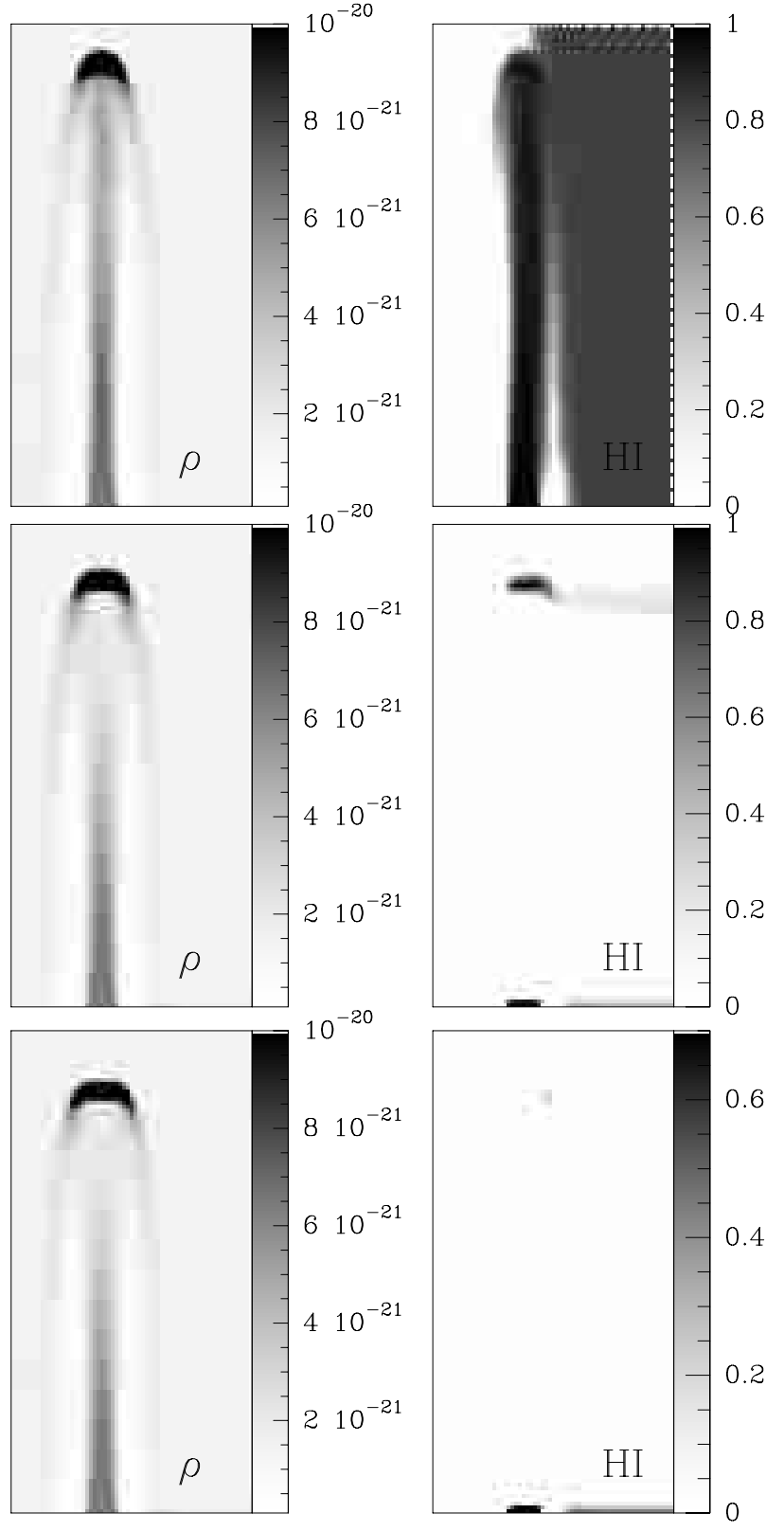

Fig. 1. Density (in $\mathrm{g} \mathrm{cm}^{-3}$, left) and neutral hydrogen fraction (right) stratifications obtained for models M1 (top), M2 (centre) and M3 (bottom) after a $t=400$ yr time-integration (see the text and Table 1). The greyscales are given by the bars to the right of each plot. The stratifications shown in this figure correspond to $x z$-cuts along the central plane of the jet, with the external ionizing photon source lying directly to the left of each plot. The plots show a $10^{17} \mathrm{~cm}$ (along the horizontal $x$-axis) by $2 \times 10^{17} \mathrm{~cm}$ (along the vertical $z$-axis) spatial domain.

optically thin, so that almost no asymmetry is introduced by the direction of propagation of the impinging ionizing photons.

From Figs. 1-4, the following effect can be seen. Models M2 and M3 have a clearly broader head than model M1. This is a direct result of the lowering of the Mach number of the jet caused by the photoionization heating of the jet beam in models M2 and M3. Because the models have a
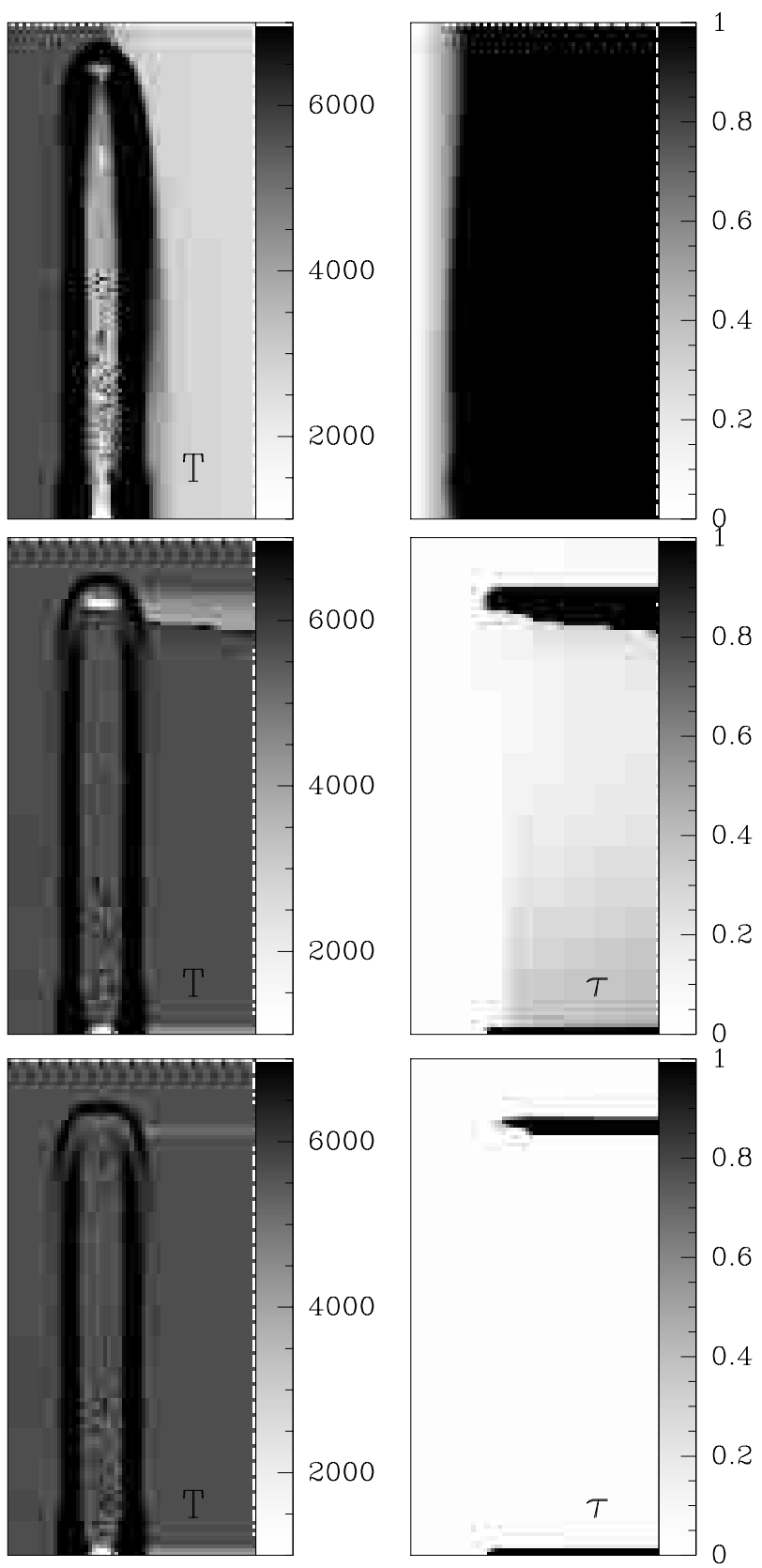

Fig. 2. Temperature (in K, left) and Lyman limit optical depth (right) stratifications obtained for models M1 (top), M2 (centre) and M3 (bottom) after a $t=400$ yr time-integration (see the text and Table 1). The greyscales are given by the bars to the right of each plot (also see Fig. 1).

highly overpressured jet beam, this lower Mach number results in a stronger lateral expansion of the beam, which then has a broader interaction region with the undisturbed environment.

A result that can be noted from the intensity maps shown in Fig. 3 is that the environment in the shadow region (which lies to the right of the working surface in model M2, see Sect. 3 and Fig. 1) does not show up as a clearly visible feature. Both the $\mathrm{H} \alpha$ and [O I] 6300 emission from this shadow region have values of $\sim 10^{-3}$ of the peak intensity of the head of the jet, so that it would be 
most difficult to detect against the background emission of the photoionized region.

Another interesting property of these models is the strong dependence of the line fluxes on the impinging photon flux $F_{*}$. Table 1 lists the peak $\mathrm{H} \alpha$ and [O I] 6300 fluxes (obtained from the intensity maps shown in Figs. 3 and 4 ). It is clear that the models with higher $F_{*}$ (models M2 and M3, see Table 1) have $\mathrm{H} \alpha$ fluxes that are a factor of $\sim 50$ larger than the $\mathrm{H} \alpha$ flux obtained for the low $F_{*}$ model M1. From this result we conclude that an $\mathrm{HH}$ object which is photoionized by an external source should show $\mathrm{H} \alpha$ fluxes (or luminosities) which are dramatically larger than the ones that would be obtained for an $\mathrm{HH}$ object located in a neutral (or molecular) region. Conversely, the [O I] 6300 line flux dramatically decreases with increasing values of the external, photoionizing flux (see Table 1).

An interesting comparison can be made between the [O I] $6300 / \mathrm{H} \alpha$ line ratio predicted for the head of the bow shock from model M1 (obtained by taking the ratio between the peak line fluxes listed for this model in Table 1) and the corresponding prediction from the planeparallel shock models of Hartigan et al. (1987). From the peak line fluxes listed for model M1 in Table 1, we obtain [O I] $6300 / \mathrm{H} \alpha=1.94$. For plane-parallel shock waves (interpolating between shock velocities and pre-shock densities in the line ratio table of Hartigan et al. 1987), we would expect a line ratio of $\approx 1.2$ for a $200 \mathrm{~km} \mathrm{~s}^{-1}$ shock wave, and a ratio of $\approx 1.9$ for a $240 \mathrm{~km} \mathrm{~s}^{-1}$ shock (both line ratios having been interpolated to a $600 \mathrm{~cm}^{-3}$ pre-shock density). Therefore, the line ratio obtained for the head of the bow shock (which has a shock velocity of $\approx 200 \mathrm{~km} \mathrm{~s}^{-1}$, see Sect. 2) is consistent in a qualitative way with predictions from plane-parallel shock models. This result gives some level of confidence in the line fluxes given in Table 1 , and indicates that these predictions are correct at least to an order of magnitude. This result is almost surprising given the rather low resolution of our numerical simulations (see Sect. 3).

\section{Conclusions}

We have computed models of a $\mathrm{HH}$ jet immersed in the photoionizing radiation emitted by an external source. From a series of three models with different values for the external, ionizing photon flux we are then able to describe the qualitative effects of the photoionization on the $\mathrm{HH}$ flow.

We find that a weak photon flux (model M1, see Table 1 and Figs. 3, 4) has the effect of enhancing the $\mathrm{H} \alpha$ emission (and reducing the [O I] 6300 emission) along the bow shock wing directed towards the ionizing photon source. We speculate that we might be observing such an effect in some of the well known HH objects such as HH 34, which shows a head with a considerably more extended (and higher excitation) Eastern bow shock wing (see, e.g., Reipurth et al. 1986; Bührke et al. 1988).
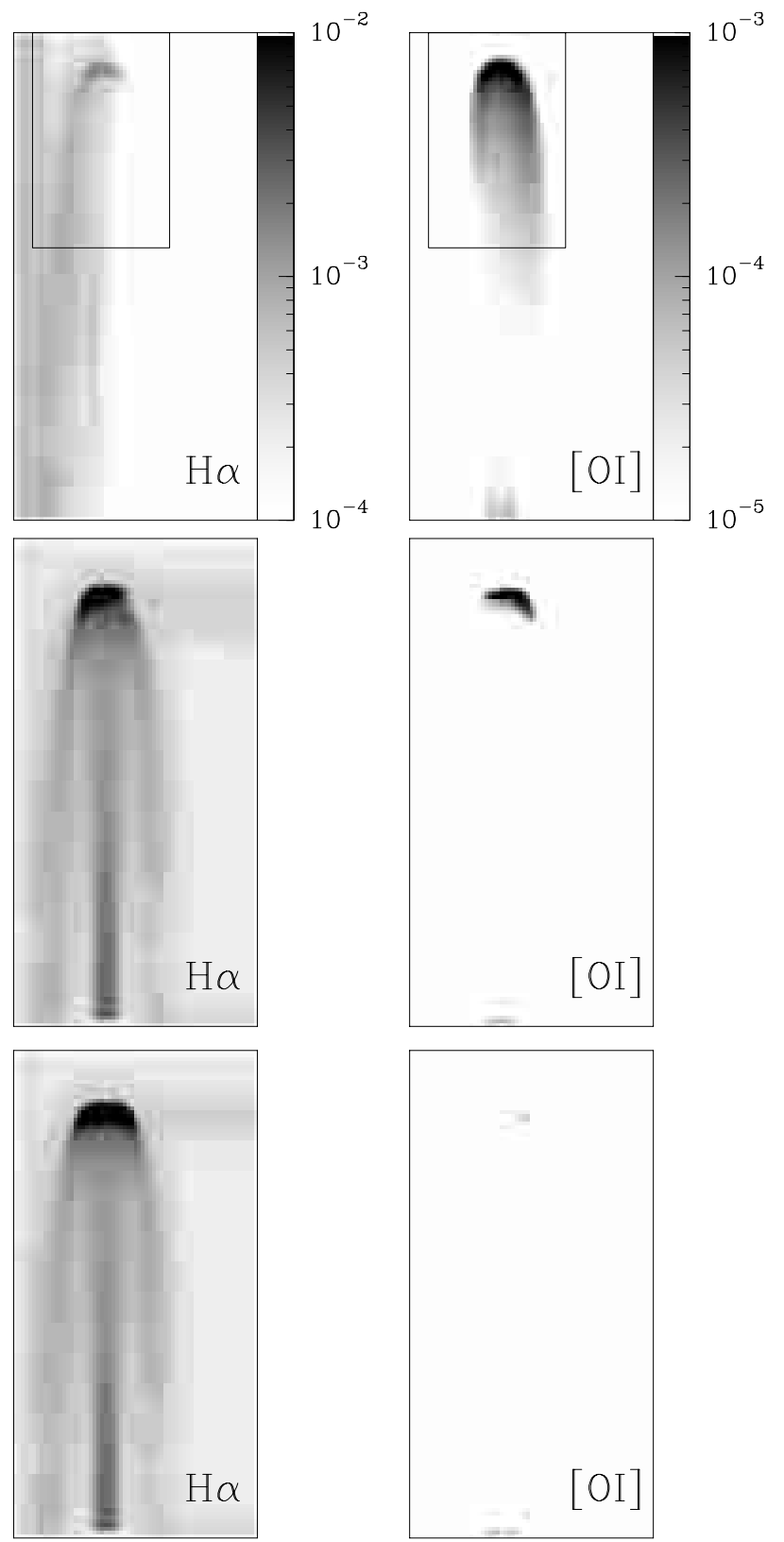

Fig. 3. $\mathrm{H} \alpha$ (left) and [O I] 6300 (right) emission line maps from models M1 (top), M2 (centre) and M3 (bottom) computed assuming that the $y$-axis is parallel to the line of sight. For more details of the plots, see the caption of Fig. 1 and the text. The three $\mathrm{H} \alpha$ maps are plotted with the same greyscale, which is depicted by the bar to the right of the top left plot (which gives the corresponding intensities in $\left.\mathrm{erg} \mathrm{s}^{-1} \mathrm{~cm}^{-2}\left({ }^{\prime \prime}\right)^{-2}\right)$. The three [O I] 6300 maps are also plotted with a single greyscale, which is depicted by the bar to the right of the top right plot.

We find that a stronger photon flux (model M2, see Table 1 and Figs. 3, 4) results in a full photoionization of the jet beam and the far bow shock wings, but the flow is still able to trap an ionization front in the head of the leading working surface. Such a configuration leads to a very clear asymmetry in the head of the jet, with stronger $\mathrm{H} \alpha$ towards the ionizing photon source and faint [O I] 6300 emission only in the opposite bow shock 

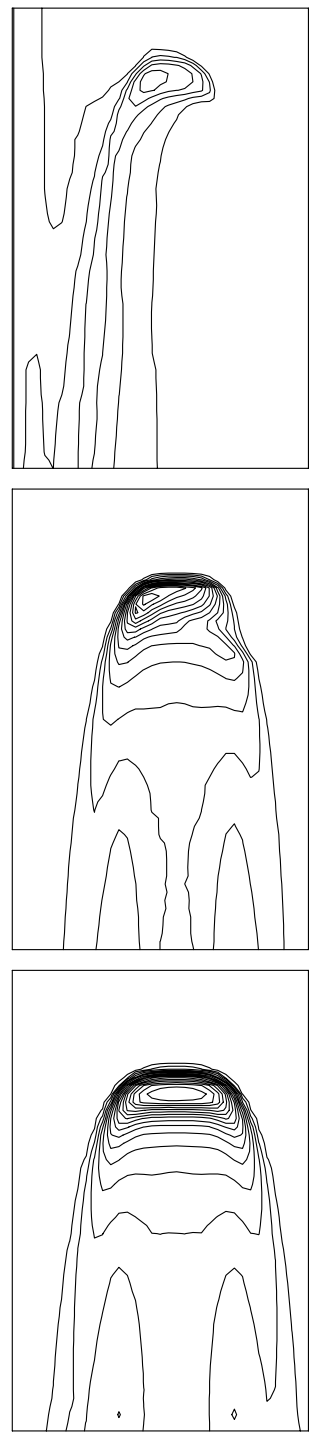

Fig. 4. Contour plots of the $\mathrm{H} \alpha$ (left) and [O I] 6300 (right) emission line maps from models M1 (top), M2 (centre) and M3 (bottom). The $\mathrm{H} \alpha$ maps are depicted with logarithmic, factor of $\sqrt{2}$ contours, and the [O I] 6300 maps are depicted with factor of 2 contours. These diagrams correspond to $5.625 \times$ $10^{16} \mathrm{~cm}$ by $8.75 \times 10^{16} \mathrm{~cm}$ regions around the working surface of the jet, which are shown as boxes in the top graphs of Fig. 3 .

wing. For even higher impinging photon fluxes (model M3, see Table 1), the head of the jet becomes almost completely symmetrical, and shows strong $\mathrm{H} \alpha$ but almost no [O I] 6300 emission.

From these results we conclude that the main indication of the existence of a trapped ionization front in an $\mathrm{HH}$ bow shock are strong, oppositely directed side to side asymmetries in $\mathrm{H} \alpha$ and in the lines of neutrals. Also, as expected, the main spectroscopic signatures of a fully photoionized $\mathrm{HH}$ object are the almost complete absence of [O I] lines and the very high $\mathrm{H} \alpha$ line fluxes (compared to "normal", i.e., not photoionized, $\mathrm{HH}$ jets).

These predictions could be compared, e.g., with the $\mathrm{HH}$ objects in the $\sigma$ Orionis region reported by Reipurth et al. (1998). HH 444 (the brightest of these HH objects) shows a very large leading working surface, with extended $\mathrm{H} \alpha$ wings. This working surface looks qualitatively similar to the broad, long-winged working surfaces predicted from our models with higher ionizing photon fluxes (models M2 and M3, see Table 1 and Fig. 4). More detailed comparisons between the models developed in the present paper and future, more detailed observations of these $\mathrm{HH}$ objects appear to be promising.

A comparison between our models and the $\mathrm{HH}$ jets in the outer regions of M 42 (reported by Bally \& Reipurth 2001) are somewhat more complex. These objects show strongly curved structures which apparently are due to the sideways interaction of the jets with the expanding H II region (also see Masciadri \& Raga 2001). In these more complex objects, it will be difficult to discern the effects of the photoionization (and the possible existence of trapped ionization fronts) from the strong asymmetries introduced by the jet/sidewind interaction.

Also, it has been pointed out by Henney (1996) that environmental density gradients can produce strong asymmetries in the bow shocks at the head of a jet. Analogously, a precession of the direction of ejection of the outflow can produce strong asymmetries in the leading bow shock (Biro et al. 1995). Therefore, given that it is possible to produce strong asymmetries in the leading bow shock in several different ways, it will not be possible to identify externally photoionized jets through such asymmetries. The more conclusive way to identify such jets then hinges on a detailed study of the emission line ratios from ionized and neutral species.

Finally, we would like to comment on the following. While our models are aimed at studying the photoionization of the leading working surface of an $\mathrm{HH}$ jet, it has now been empirically shown that many of which were thought to be the "heads" of $\mathrm{HH}$ jets actually turn out to be "internal working surfaces" which have been preceeded by other previous ejection events. This has been quite dramatically shown by the discovery of "super jets" associated with many of the previously known $\mathrm{HH}$ jets, for example with HH 34 (Bally \& Devine 1994; Devine et al. 1997; Eislöffel \& Mundt 1997) and HH 111 (Reipurth et al. 1997). Actually, it is non-trivial to identify which (if any) of the observed knots actually is the leading working surface of the jet (Rosado et al. 1999).

On the other hand, our models refer to the photoionization of the "leading working surface" of a jet, and not to an internal working surface. We have chosen this problem because it has many fewer free parameters, and is therefore easier to explore numerically. Previous numerical simulations (Raga \& Noriega-Crespo 1998; Völker et al. 1999; de Gouveia dal Pino 2001) have shown that internal working surfaces resulting from ejection velocity variabilities with large amplitudes (i.e., with amplitudes comparable to the mean value of the ejection velocity) strongly resemble the leading working surface of the jet. Such variabilities are actually necessary for reproducing the large bow shocks observed along $\mathrm{HH}$ jets (such as the $\mathrm{HH} 34 \mathrm{~S}$ bow shock, see Raga \& Noriega-Crespo 1998). The models 
that we have presented above would therefore also apply (at least in a qualitative way) to the photoionization of such "large" internal working surfaces along HH jets.

Acknowledgements. This work was supported by the CONACyT grant 32753-E and the DGAPA (UNAM) grant IN-128098. A. R. acknowledges support from a fellowship of the John Simon Guggenheim Foundation. We thank an anonymous referee for helpful comments.

\section{References}

Bally, J., \& Devine, D. 1994, ApJ, 428, L65

Bally, J., O'Dell, C. R., \& McCaughrean, M. J. 2000, AJ, 119, 2919

Bally, J., \& Reipurth, B. 2001, ApJ, 546, 299

Biro, S., Raga, A. C., \& Cantó, J. 1995, MNRAS, 275, 557

Bührke, T., Mundt, R., \& Ray, T. P. 1988, A\&A, 200, 99

Cantó J., Raga, A. C., Steffen W., \& Shapiro P. 1998, ApJ, 502,695

Devine, D., Bally, J., Reipurth, B., \& Heathcote, S. 1997, AJ, 114, 2095

Eislöffel, J., \& Mundt, R. 1997, AJ, 114, 280

de Gouveia dal Pino, E. M. 2001, ApJ, 551, 347
Hartigan, P., Raymond, J. C., \& Hartmann, L. W. 1987, ApJ, 316,323

Henney, W. J. 1996, RMxAA, 32, 3

Masciadri, E., \& Raga, A. C. 2001, AJ, 121, 408

Pavlakis, K. G., Williams, R. J. R., Dyson, J. E., Falle, S. A. E. G., \& Hartquist, T. W. 2001, A\&A, 369, 263

Raga, A. C., Noriega-Crespo, A., Cantó, J., et al. 1997, RMxAA, 33, 73

Raga, A. C., \& Noriega-Crespo, A. 1998, AJ, 116, 2943

Raga, A. C., Mellema, G., Arthur, S. J., et al. 1999, RMxAA, 35,123

Raga, A. C., López-Martin, L., Binette, L., et al. 2000a, MNRAS, 314, 681

Raga, A. C., Navarro-González, R., \& Villagrán-Muniz, M. 2000b, RMxAA, 36, 67

Raga, A. C., Cabrit, S., Dougados, C., \& Lavalley, C. 2001, ApJ, 548, 288

Reipurth, B., Bally, J., Graham, J. A., Lane, A. P., \& Zealy, W. J. 1986, A\&A, 164, 61

Reipurth, B., Bally, J., \& Devine, D. 1997, AJ, 114, 2708

Reipurth, B., Bally, J., Fesen, R. A., \& Devine D. 1998, Nature, 396,343

Rosado, M., Raga, A. C., \& Arias, L. 1999, AJ, 117, 462

Van Leer, B. 1982, ICASE Report, No. 82-30

Völker, R., Smith, M. D., Suttner, G., \& Yorke, H. W. 1999, A\&A, 343, 953 